\title{
Nonlinear Constraints on Relativistic Fluids Far from Equilibrium
}

\author{
Fábio S. Bemfica๑, ${ }^{1, *}$ Marcelo M. Disconzi $\odot,{ }^{2, \dagger}$ Vu Hoang $\odot,{ }^{3, \$}$ Jorge Noronha, ${ }^{4,}$ and Maria Radosz ${ }^{3, \|}$ \\ ${ }^{1}$ Escola de Ciências e Tecnologia, Universidade Federal do Rio Grande do Norte, 59072-970, Natal, Rio Grande do Norte, Brazil \\ ${ }^{2}$ Department of Mathematics, Vanderbilt University, Nashville, Tennessee 37211, USA \\ ${ }^{3}$ Department of Mathematics, The University of Texas at San Antonio, One UTSA Circle, San Antonio, Texas 78249, USA \\ ${ }^{4}$ Illinois Center for Advanced Studies of the Universe, Department of Physics, \\ University of Illinois at Urbana-Champaign, Urbana, Illinois 61801, USA
}

(Received 6 June 2020; revised 6 March 2021; accepted 29 April 2021; published 1 June 2021)

\begin{abstract}
New constraints are found that must necessarily hold for Israel-Stewart-like theories of fluid dynamics to be causal far away from equilibrium. Conditions that are sufficient to ensure causality, local existence, and uniqueness of solutions in these theories are also presented. Our results hold in the full nonlinear regime, taking into account bulk and shear viscosities (at zero chemical potential), without any simplifying symmetry or near-equilibrium assumptions. Our findings provide fundamental constraints on the magnitude of viscous corrections in fluid dynamics far from equilibrium.
\end{abstract}

DOI: 10.1103/PhysRevLett.126.222301

Introduction.-Relativistic fluid dynamics is essential to the state-of-the-art modeling of the quark-gluon plasma (QGP) formed in ultrarelativistic heavy-ion collisions (see [1-3]). However, despite its wide use and significant success, it remains unclear why such a fluid dynamical description is applicable given that local deviations from equilibrium in nucleus-nucleus collisions can be very large, especially at early times [4-6]. In fact, typical fluidlike signatures involving anisotropic flow [7] persist even in small systems formed in proton-nucleus and proton-proton collisions at sufficiently high multiplicity [8-14]. Such findings have motivated a series of new investigations on the foundations of relativistic viscous fluid dynamics [15-18] and their subsequent extension toward the far-from-equilibrium regime relevant for heavy-ion collisions [19-45].

The viscous fluid description of the QGP is currently based on ideas from Israel and Stewart (IS) [46,47] (see, also, Mueller [48]), who proposed a way to fix the long-standing acausality [49] and instability [50] problems of the relativistic generalization of Navier-Stokes (NS) equations derived by Eckart [51] and Landau and Lifshitz [52]. The general mechanism introduced by IS to try to avoid such issues assumes that dissipative currents such as the shear stress tensor, $\pi_{\mu \nu}$, and the bulk scalar, $\Pi$, are new degrees of freedom $[53,54]$ which obey nonlinear relaxation equations describing how such quantities relax to their relativistic NS limits within relaxation time scales $\tau_{\pi}$ and $\tau_{\Pi}$. The same principle is also at

Published by the American Physical Society under the terms of the Creative Commons Attribution 4.0 International license. Further distribution of this work must maintain attribution to the author(s) and the published article's title, journal citation, and DOI. Funded by SCOAP. play in modern formulations of fluid dynamics put forward by Ref. [55] and Ref. [56], which are currently employed in numerical simulations (see, for instance, [57]).

It is well known that the IS-like theories are linearly stable around equilibrium [58-61]. But physically sensible relativistic theories of fluid dynamics must also be causal, i.e., the equations of motion must be hyperbolic, and the propagation of information must be, at most, the speed of light [62]. Also, the Cauchy problem must be locally well posed [63], i.e., given initial conditions, one must show that the equations admit a unique solution. A common misconception in the field is that IS-like theories have already been proven to be causal a long time ago in Refs. [46,58,59,64-66]. This is not the case. Those early works only considered linearized disturbances around equilibrium, where the background fields $\pi_{\mu \nu}$ and $\Pi$ vanish and the corresponding linear disturbances are small. Such a linearized analysis says nothing about the nonlinear regime, even for small $\pi_{\mu \nu}$ and $\Pi$. The far-from-equilibrium regime, in particular, is necessarily nonlinear as $\pi_{\mu \nu}$ and $\Pi$ can be as large as the local equilibrium pressure $P$.

Hence, it is not known if IS theories are, indeed, sensible in the regime probed by high energy hadronic collisions. Understanding the far-from-equilibrium properties of such theories is also crucial to reliably assess the role of viscous effects in early universe cosmology [67]. Here, we make essential steps toward solving this critical problem by finding conditions (in the form of simple algebraic inequalities that can be checked at every step of the evolution) that must necessarily hold for IS-like theories to be causal in the nonlinear regime. We also present conditions that are sufficient to ensure causality, local existence, and uniqueness of solutions of IS-like theories. Our results are the first in the literature that hold in the full nonlinear regime, with 
bulk and shear viscosities (at zero chemical potential), in three spatial dimensions, without recurring to any symmetry or near-equilibrium assumptions.

The equations of motion.-Using the Landau frame definition of the hydrodynamic variables [52], the energy-momentum tensor of the fluid can be written as [We use units $c=\hbar=k_{B}=1$. The spacetime metric signature is $(-+++)$. Greek indices run from 0 to 3 , Latin indices from 1 to 3.] $T^{\mu \nu}=\varepsilon u^{\mu} u^{\nu}+$ $(P+\Pi) \Delta^{\mu \nu}+\pi^{\mu \nu}$, where $u^{\mu}$ is the fluid's four-velocity (with $u_{\mu} u^{\mu}=-1$ ), $\varepsilon$ is the energy density, $P=P(\varepsilon)$ is the equilibrium pressure defined by an equation of state, $\Delta_{\mu \nu}=g_{\mu \nu}+u_{\mu} u_{\nu}$ is the projector orthogonal to the flow, $g_{\mu \nu}$ is the spacetime metric, $\pi_{\mu \nu}=\pi_{\nu \mu}, \pi^{\mu \nu} u_{\mu}=0$, and $\Delta_{\mu \nu} \pi^{\mu \nu}=0$. We focus on high energy collisions, and thus, we only investigate, here, the case of zero chemical potentials. Conservation of energy and momentum implies that $\nabla_{\mu} T^{\mu \nu}=0$, which can be written as $\left(c_{s}^{2}=d P / d \varepsilon\right.$ is the equilibrium speed of sound squared)

$$
\begin{aligned}
& u^{\alpha} \nabla_{\alpha} \varepsilon+(\varepsilon+P+\Pi) \nabla_{\alpha} u^{\alpha}+\pi_{\mu}^{\alpha} \nabla_{\alpha} u^{\mu}=0, \\
&(\varepsilon+P+\Pi) u^{\beta} \nabla_{\beta} u_{\alpha}+c_{s}^{2} \Delta_{\alpha}^{\beta} \nabla_{\beta} \varepsilon+\Delta_{\alpha}^{\beta} \nabla_{\beta} \Pi+\Delta_{\alpha}^{\beta} \nabla_{\mu} \pi_{\beta}^{\mu}=0 .
\end{aligned}
$$

Here, we consider the case where the dissipative currents $\left\{\pi^{\mu \nu}, \Pi\right\}$ satisfy the following equations (Note that our metric signature is different than in [56].), derived using the Denicol-Niemi-Molnar-Rischke formalism [56], and commonly used in heavy-ion collision applications

$$
\tau_{\Pi} u^{\mu} \nabla_{\mu} \Pi+\Pi=-\zeta \nabla_{\mu} u^{\mu}-\delta_{\Pi \Pi} \Pi \nabla_{\mu} u^{\mu}-\lambda_{\Pi \pi} \pi^{\mu \nu} \sigma_{\mu \nu},
$$

$$
\begin{aligned}
& \tau_{\pi} \Delta_{\alpha \beta}^{\mu \nu} u^{\lambda} \nabla_{\lambda} \pi^{\alpha \beta}+\pi^{\mu \nu} \\
& \quad=-2 \eta \sigma^{\mu \nu}-\delta_{\pi \pi} \pi^{\mu \nu} \nabla_{\alpha} u^{\alpha}-\tau_{\pi \pi} \pi_{\alpha}^{\langle\mu} \sigma^{\nu\rangle \alpha}-\lambda_{\pi \Pi} \Pi \sigma^{\mu \nu},
\end{aligned}
$$

where $\sigma^{\mu \nu}=\Delta_{\alpha \beta}^{\mu \nu} \nabla^{\alpha} u^{\beta}$ is the shear tensor, $\Delta_{\alpha \beta}^{\mu \nu}=$ $\left(\Delta_{\alpha}^{\mu} \Delta_{\beta}^{\nu}+\Delta_{\beta}^{\mu} \Delta_{\alpha}^{\nu}\right) / 2-\frac{1}{3} \Delta^{\mu \nu} \Delta_{\alpha \beta}, A_{\lambda}^{\langle\mu} B^{\nu \lambda \lambda}=\Delta_{\alpha \beta}^{\mu \nu} A^{\alpha \lambda} B_{\lambda}^{\beta}$, and $\eta, \zeta$ are the shear and bulk viscosities, respectively. All the transport coefficients, $\left\{\eta, \zeta, \tau_{\Pi}, \tau_{\pi}, \delta_{\Pi \Pi}, \lambda_{\Pi \pi}, \delta_{\pi \pi}, \tau_{\pi \pi}, \lambda_{\pi \Pi}\right\}$, can depend on the ten dynamical variables $\left\{\varepsilon, u_{\mu}, \pi_{\mu \nu}, \Pi\right\}$ (so, in principle, they may even depend on the dissipative tensors) but not on their derivatives. Explicit expressions for transport coefficients in models can be found, for instance, in $[56,68,69]$.

We note that $\left\{\eta, \zeta, \tau_{\pi}, \tau_{\Pi}\right\}$ are the only coefficients that remain after linearization around equilibrium where $\pi^{\mu \nu}=0$ and $\Pi=0$. This shows why linearized analyses [58,59] necessarily miss the effects from the other coefficients, $\left\{\delta_{\Pi \Pi}, \lambda_{\Pi \pi}, \delta_{\pi \pi}, \tau_{\pi \pi}, \lambda_{\pi \Pi}\right\}$, which contribute to the nonlinear evolution. However, other nonlinear terms such as $\pi_{\mu \nu} \pi^{\mu \nu}, \Pi^{2}, \pi^{\mu \nu} \Pi, \pi_{\alpha}^{\langle\mu} \pi^{\nu\rangle \alpha}$, which appear in [56], could have been trivially added to the equations as they do not contribute to a causality analysis since they do not involve derivatives of the fields. Nevertheless, there are still some other nonlinear terms that can be considered such as $\pi_{\alpha}^{\langle\mu} \Omega^{\nu\rangle \alpha}$, where $\Omega_{\mu \nu}=\left(\Delta_{\mu}^{\alpha} \nabla_{\alpha} u_{\nu}-\right.$ $\left.\Delta_{\nu}^{\alpha} \nabla_{\alpha} u_{\mu}\right) / 2$ is the vorticity and, also, $\Omega_{\alpha}^{\langle\mu} \Omega^{\nu\rangle \alpha}$ [3]. The former will be investigated in a separate publication. The latter contributes with derivatives of the fields to the principal part of the system of equations and, thus, a different analysis than presented here would be required.

Causality.-Causality is the concept in relativity theory asserting that no information propagates faster than the speed of light and no closed timelike curves exist (so the future cannot influence the past). See Refs. [70-74] for a mathematically precise definition of causality. Causality can be investigated by determining the characteristic manifolds associated with a system of partial differential equations [75,76]. Let us write equations (1)-(2) as $A^{\alpha} \nabla_{\alpha} \Psi=F(\Psi)$, where we defined the vector $\Psi=\left(\varepsilon, u^{\nu}, \Pi, \pi^{0 \nu}, \pi^{1 \nu}, \pi^{2 \nu}, \pi^{3 \nu}\right)$, the $22 \times 22$ matrix

$$
A^{\alpha}=\left[\begin{array}{ccccccc}
u^{\alpha} & \rho \delta_{\nu}^{\alpha}+\pi_{\nu}^{\alpha} & 0_{1 \times 1} & 0_{1 \times 4} & 0_{1 \times 4} & 0_{1 \times 4} & 0_{1 \times 4} \\
c_{s}^{2} \Delta^{\mu \alpha} & \rho u^{\alpha} \delta_{\nu}^{\mu}-\pi_{\nu}^{\alpha} u^{\mu} & \Delta^{\mu \alpha} & \delta_{0}^{\alpha} I_{4} & \delta_{1}^{\alpha} I_{4} & \delta_{2}^{\alpha} I_{4} & \delta_{3}^{\alpha} I_{4} \\
0_{4 \times 1} & E_{\nu}^{\alpha} & \tau_{\Pi} u^{\alpha} & 0_{4 \times 4} & 0_{4 \times 4} & 0_{4 \times 4} & 0_{4 \times 4} \\
0_{4 \times 1} & C_{\nu}^{0 \delta \alpha} & 0_{4 \times 1} & \tau_{\pi} u^{\alpha} I_{4} & 0_{4 \times 4} & 0_{4 \times 4} & 0_{4 \times 4} \\
0_{4 \times 1} & C_{\nu}^{1 \delta \alpha} & 0_{4 \times 1} & 0_{4 \times 4} & \tau_{\pi} u^{\alpha} I_{4} & 0_{4 \times 4} & 0_{4 \times 4} \\
0_{4 \times 1} & C_{\nu}^{2 \delta \alpha} & 0_{4 \times 1} & 0_{4 \times 4} & 0_{4 \times 4} & \tau_{\pi} u^{\alpha} I_{4} & 0_{4 \times 4} \\
0_{4 \times 1} & C_{\nu}^{3 \delta \alpha} & 0_{4 \times 1} & 0_{4 \times 4} & 0_{4 \times 4} & 0_{4 \times 4} & \tau_{\pi} u^{\alpha} I_{4}
\end{array}\right],
$$


and $F(\Psi)$ is a vector that does not contain derivatives of the variables. Above, we also defined $\rho=\varepsilon+P+\Pi$, $E_{\nu}^{\alpha}=\left(\zeta+\delta_{\Pi \Pi} \Pi\right) \delta_{\nu}^{\alpha}+\lambda_{\Pi \pi} \pi_{\nu}^{\alpha}, \quad B_{\nu}^{\mu \lambda \alpha}=\frac{1}{2}\left(\Delta^{\mu \alpha} \delta_{\nu}^{\lambda}+\Delta^{\lambda \alpha} \delta_{\nu}^{\mu}\right.$ $\left.-\frac{2}{3} \Delta^{\mu \lambda} \delta_{\nu}^{\alpha}\right)$, and

$$
\begin{aligned}
C_{\nu}^{\sigma \delta \alpha}= & {\left[\left(2 \eta+\lambda_{\pi \Pi} \Pi\right) \delta_{\mu}^{\sigma} \delta_{\lambda}^{\delta}+\frac{\tau_{\pi \pi}}{2} \pi_{\lambda}^{\sigma} \delta_{\mu}^{\delta}+\frac{\tau_{\pi \pi}}{2} \pi_{\lambda}^{\delta} \delta_{\mu}^{\sigma}\right] B_{\nu}^{\mu \lambda \alpha} } \\
& -\frac{\tau_{\pi \pi}}{3} \Delta^{\sigma \delta} \pi_{\nu}^{\alpha}+\delta_{\pi \pi} \pi^{\sigma \delta} \delta_{\nu}^{\alpha}-\tau_{\pi}\left(\pi_{\nu}^{\sigma} u^{\delta}+\pi_{\nu}^{\delta} u^{\sigma}\right) u^{\alpha} .
\end{aligned}
$$

The characteristic surfaces $\{\Phi(x)=0\}$ are determined by the principal part of the equations by solving the characteristic equation $\operatorname{det}\left(A^{\alpha} \xi_{\alpha}\right)=0$, with $\xi_{\alpha}=\nabla_{\alpha} \Phi$ [77]. The system is causal if, for any $\xi_{i}$, it holds that (C1) the roots $\xi_{0}=\xi_{0}\left(\xi_{i}\right)$ of the characteristic equation are real and (C2) $\xi_{\alpha}=\left(\xi_{0}, \xi_{i}\right)$ is spacelike or lightlike. Condition (C2) implies that the characteristic surfaces $\{\Phi(x)=0\}$ are timelike or lightlike, indicating that no information is superluminal. For instance, for an ideal fluid (where $\Pi=0$ and $\pi_{\mu \nu}=0$ ), the characteristic velocities are determined by the speed of sound and causality implies that $c_{s}^{2} \leq 1$ [63].

From (3), it is clear that the characteristics associated with the evolution depend on the dissipative tensors $\left\{\pi^{\mu \nu}, \Pi\right\}$. Therefore, the true causal behavior of IS theories is necessarily a far-from-equilibrium property of the fluid, and linear analyses around equilibrium cannot be used to establish causality and well posedness in IS theories. The computation of the characteristics defined by (3), which is needed for a causality analysis, is extremely involved and is presented in the Supplemental Material [78]. Below, we present the main consequences of such calculations.

Let $\Lambda_{\alpha}, \alpha=0,1,2,3$, be the eigenvalues of the $\pi_{\nu}^{\mu}$. The eigenvalues are such that $\Lambda_{0}=0$, since $u_{\mu}$ is in the kernel of $\pi_{\nu}^{\mu}\left(u_{\mu} \pi_{\nu}^{\mu}=0\right)$, and $\Lambda_{1}+\Lambda_{2}+\Lambda_{3}=0$, so that the trace is kept zero. Without loss of generality, let us take $\Lambda_{1} \leq \Lambda_{2} \leq \Lambda_{3}$ with $\Lambda_{1} \leq 0 \leq \Lambda_{3}$. Now, we state our assumptions, which are the following: (A1) for the transport coefficients and relaxation times, suppose that $\tau_{\Pi}, \tau_{\pi}>$ 0 and $\eta, \zeta, \tau_{\pi \pi}, \delta_{\Pi \Pi}, \lambda_{\Pi \pi}, \delta_{\pi \pi}, \lambda_{\pi \Pi}, c_{s}^{2} \geq 0$; (A2) for the fluid variables, suppose that $\varepsilon>0, P \geq 0$, and $\varepsilon+P+\Pi>0$; finally, we also assume that (A3) $\varepsilon+P+\Pi+\Lambda_{a}>0$, $a=1,2,3$. Then, the following conditions are necessary for causality, i.e., if any of the inequalities below is not satisfied, then the system is not causal:

$$
\begin{gathered}
\left(2 \eta+\lambda_{\pi \Pi} \Pi\right)-\frac{1}{2} \tau_{\pi \pi}\left|\Lambda_{1}\right| \geq 0, \\
\varepsilon+P+\Pi-\frac{1}{2 \tau_{\pi}}\left(2 \eta+\lambda_{\pi \Pi} \Pi\right)-\frac{\tau_{\pi \pi}}{4 \tau_{\pi}} \Lambda_{3} \geq 0, \\
\frac{1}{2 \tau_{\pi}}\left(2 \eta+\lambda_{\pi \Pi} \Pi\right)+\frac{\tau_{\pi \pi}}{4 \tau_{\pi}}\left(\Lambda_{a}+\Lambda_{d}\right) \geq 0, \quad a \neq d,
\end{gathered}
$$

$$
\begin{gathered}
\varepsilon+P+\Pi+\Lambda_{a}-\frac{1}{2 \tau_{\pi}}\left(2 \eta+\lambda_{\pi \Pi} \Pi\right) \\
-\frac{\tau_{\pi \pi}}{4 \tau_{\pi}}\left(\Lambda_{d}+\Lambda_{a}\right) \geq 0, \quad a \neq d, \\
\frac{1}{2 \tau_{\pi}}\left(2 \eta+\lambda_{\pi \Pi} \Pi\right)+\frac{\tau_{\pi \pi}}{2 \tau_{\pi}} \Lambda_{d} \\
+\frac{1}{6 \tau_{\pi}}\left[2 \eta+\lambda_{\pi \Pi} \Pi+\left(6 \delta_{\pi \pi}-\tau_{\pi \pi}\right) \Lambda_{d}\right] \\
+\frac{\zeta+\delta_{\Pi \Pi} \Pi+\lambda_{\Pi \pi} \Lambda_{d}}{\tau_{\Pi}}+\left(\varepsilon+P+\Pi+\Lambda_{d}\right) c_{s}^{2} \geq 0, \\
\varepsilon+P+\Pi+\Lambda_{d}-\frac{1}{2 \tau_{\pi}}\left(2 \eta+\lambda_{\pi \Pi} \Pi\right) \\
-\frac{\tau_{\pi \pi}}{2 \tau_{\pi}} \Lambda_{d}-\frac{1}{6 \tau_{\pi}}\left[2 \eta+\lambda_{\pi \Pi} \Pi+\left(6 \delta_{\pi \pi}-\tau_{\pi \pi}\right) \Lambda_{d}\right] \\
-\frac{\zeta+\delta_{\Pi \Pi} \Pi+\lambda_{\Pi \pi} \Lambda_{d}}{\tau_{\Pi}}-\left(\varepsilon+P+\Pi+\Lambda_{d}\right) c_{s}^{2} \geq 0,
\end{gathered}
$$

where (4c)-(4f) must hold for $a, d=1,2,3$. The proof that (4) are necessary conditions for causality under assumptions (A1)-(A3) is given in the Supplemental Material [78]. Here, we discuss the significance of this result.

We stress that assumptions (A1) and (A2) are standard in heavy-ion collision applications [57], and (A3) is a very natural assumption since $P+\Pi+\Lambda_{a}$ for $a=1,2,3$ may be interpreted as the pressure in each spatial axis in the local rest frame. Thus, if (A3) is violated, the theory would have a pathology in the sense that fluid elements would have negative inertia, i.e., the acceleration is opposite to the force given by the negative of the gradient of pressure. Furthermore, it is natural to make assumptions that hold close to equilibrium, and since (A2) guarantees $\varepsilon+P+\Pi>0$, for small deviations from equilibrium, $\Lambda_{a}$ will be small, giving $\varepsilon+P+\Pi+\Lambda_{a}>0$. That said, we stress that, although (A3) is expected to hold near equilibrium, it is, itself, not a near-equilibrium assumption.

Conditions (4) could never have been found using a linearized analysis, as they depend on $\Pi$ and $\Lambda_{a}$, both of which vanish in equilibrium. Consequently, if, in any fluid dynamic simulation in heavy-ion collisions that employs (1)-(2), the necessary conditions above are not fulfilled, causality is necessarily violated. It is important to point out that this causality violation has nothing to do with the ability of numerical schemes to produce a solution, a point we shall return to at the end of the Letter.

While the above conditions must hold for the system to be causal, they are not sufficient conditions, i.e., by themselves, conditions (A1)-(A3) and (4) do not assure the system to be causal (see the Supplemental Material 
[78]). Therefore, it is important to have conditions that are sufficient for causality. In this regard, assume, again, that (A1)-(A3) hold. Then, the following conditions are sufficient to ensure that causality holds, i.e., if they are satisfied, then the system is causal:

$$
\begin{gathered}
\left(\varepsilon+P+\Pi-\left|\Lambda_{1}\right|\right)-\frac{1}{2 \tau_{\pi}}\left(2 \eta+\lambda_{\pi \Pi} \Pi\right)-\frac{\tau_{\pi \pi}}{2 \tau_{\pi}} \Lambda_{3} \geq 0, \\
\left(2 \eta+\lambda_{\pi \Pi} \Pi\right)-\tau_{\pi \pi}\left|\Lambda_{1}\right|>0, \\
\tau_{\pi \pi} \leq 6 \delta_{\pi \pi}, \\
\frac{\lambda_{\Pi \pi}}{\tau_{\Pi}}+c_{s}^{2}-\frac{\tau_{\pi \pi}}{12 \tau_{\pi}} \geq 0,
\end{gathered}
$$$$
\frac{1}{3 \tau_{\pi}}\left[4 \eta+2 \lambda_{\pi \Pi} \Pi+\left(3 \delta_{\pi \pi}+\tau_{\pi \pi}\right) \Lambda_{3}\right]
$$$$
+\frac{\zeta+\delta_{\Pi \Pi} \Pi+\lambda_{\Pi \pi} \Lambda_{3}}{\tau_{\Pi}}+\left|\Lambda_{1}\right|+\Lambda_{3} c_{s}^{2}
$$$$
+\frac{\frac{12 \delta_{\pi \pi}-\tau_{\pi \pi}}{12 \tau_{\pi}}\left(\frac{\lambda_{\Pi \pi}}{\tau_{\Pi}}+c_{s}^{2}-\frac{\tau_{\pi \pi}}{12 \tau_{\pi}}\right)\left(\Lambda_{3}+\left|\Lambda_{1}\right|\right)^{2}}{\varepsilon+P+\Pi-\left|\Lambda_{1}\right|-\frac{1}{2 \tau_{\pi}}\left(2 \eta+\lambda_{\pi \Pi} \Pi\right)-\frac{\tau_{\pi \pi}}{2 \tau_{\pi}} \Lambda_{3}}
$$$$
\leq(\varepsilon+P+\Pi)\left(1-c_{s}^{2}\right) \text {, }
$$$$
\frac{1}{6 \tau_{\pi}}\left[2 \eta+\lambda_{\pi \Pi} \Pi+\left(\tau_{\pi \pi}-6 \delta_{\pi \pi}\right)\left|\Lambda_{1}\right|\right]
$$$$
+\frac{\zeta+\delta_{\Pi \Pi} \Pi-\lambda_{\Pi \pi}\left|\Lambda_{1}\right|}{\tau_{\Pi}}+\left(\varepsilon+P+\Pi-\left|\Lambda_{1}\right|\right) c_{s}^{2} \geq 0,
$$

$$
1 \geq \frac{\frac{12 \delta_{\pi \pi}-\tau_{\pi \pi}}{12 \tau_{\pi}}\left(\frac{\lambda_{\Pi \pi}}{\tau_{\Pi}}+c_{s}^{2}-\frac{\tau_{\pi \pi}}{12 \tau_{\pi}}\right)\left(\Lambda_{3}+\left|\Lambda_{1}\right|\right)^{2}}{\left[\frac{1}{2 \tau_{\pi}}\left(2 \eta+\lambda_{\pi \Pi} \Pi\right)-\frac{\tau_{\pi \pi}}{2 \tau_{\pi}}\left|\Lambda_{1}\right|\right]^{2}},
$$

$$
\begin{aligned}
& \frac{1}{3 \tau_{\pi}}\left[4 \eta+2 \lambda_{\pi \Pi} \Pi-\left(3 \delta_{\pi \pi}+\tau_{\pi \pi}\right)\left|\Lambda_{1}\right|\right] \\
& \quad+\frac{\zeta+\delta_{\Pi \Pi} \Pi-\lambda_{\Pi \pi}\left|\Lambda_{1}\right|}{\tau_{\Pi}}+\left(\varepsilon+P+\Pi-\left|\Lambda_{1}\right|\right) c_{s}^{2} \\
& \quad \geq \frac{\left(\varepsilon+P+\Pi+\Lambda_{2}\right)\left(\varepsilon+P+\Pi+\Lambda_{3}\right)}{3\left(\varepsilon+P+\Pi-\left|\Lambda_{1}\right|\right)} \\
& \quad \times\left\{1+\frac{2\left[\frac{1}{2 \tau_{\pi}}\left(2 \eta+\lambda_{\pi \Pi} \Pi\right)+\frac{\tau_{\pi \pi}}{2 \tau_{\pi}} \Lambda_{3}\right]}{\varepsilon+P+\Pi-\left|\Lambda_{1}\right|}\right\},
\end{aligned}
$$

where condition (5h) can be dropped if $\delta_{\pi \pi}=\tau_{\pi \pi}=0$. The detailed proof can be found in the Supplemental Material [78]. Since (4) must hold for causality, they must be satisfied for any set of conditions that imply causality, and it is possible to verify that (5) imply (4) under assumptions (A1)-(A3). When shear viscous effects are neglected, (5) reduces to the conditions for the bulk viscosity case found in [79].
Conditions (A1)-(A3) and (5) also ensure the unique local solvability of the initial-value problem in the class of quasianalytic functions: given initial data of sufficient regularity satisfying (5), there exists a unique solution to the nonlinear equations taking the given initial data, defined for a certain time interval. Thus, if (A1)-(A3) and (5) hold, the evolution of the viscous fluid is guaranteed to be well defined and causal even far from equilibrium where the gradients (and, hence, $\pi^{\mu \nu}$ and $\Pi$ ) are large. This is relevant for determining the properties of hydrodynamic attractors [20] under general flow conditions $[27,80]$ and, also, for an overall validation of fluid dynamics descriptions of extreme systems, such as proton-proton collisions. Moreover, while, here, we focus on applications to heavy-ion collisions, so $g_{\mu \nu}$ is the Minkowski metric, the methods of [79] can be adapted to show that our conclusions hold when (1)-(2) are coupled to Einstein's equations. Hence, our results are also crucial for determining the far-from-equilibrium behavior of viscous fluids in general relativity, which may be relevant to neutron star mergers [81]. The technical details of these statements are provided in the Supplemental Material [78].

When we linearize the equations around the equilibrium, terms involving $\tau_{\pi \pi}, \delta_{\Pi \Pi}, \lambda_{\Pi \pi}, \delta_{\pi \pi}, \lambda_{\pi \Pi}$ drop out and, thus, (A1) can be replaced by $\tau_{\pi}, \tau_{\Pi}>0, \eta, \zeta, c_{s}^{2} \geq 0$, and (A2) and (A3) can be replaced by $\varepsilon+P>0$ and $P \geq 0$. Then, conditions (5) become necessary and reduce to $\varepsilon+P>0$, $\varepsilon+P-\left(\eta / \tau_{\pi}\right) \geq 0, \quad$ and $\quad 1 /(\varepsilon+P)\left(4 \eta / 3 \tau_{\pi}+\zeta / \tau_{\Pi}\right) \leq$ $1-c_{s}^{2}$. These conditions coincide with the corresponding well-known results previously found in $[58,59]$ that ensure causality and stability in the linearized regime around equilibrium.

It is instructive to compare the causal propagation modes of the full nonlinear theory determined here with that of the dynamics linearized about equilibrium. Linearizing Eqs. (1)-(2) around equilibrium, we find four distinct modes of propagation which correspond to the flow lines, the sound waves, and shear waves at two distinct speeds. These are the same ones found in the previous works $[46,58,59,64-66]$, where, there, the authors find, in addition, a second longitudinal mode (second sound) due to the fact that their equations also include a conserved current. In the nonlinear case, we found six distinct propagation modes corresponding to the flow lines, the sound waves, and shear waves at four distinct speeds. This, again, highlights how one misses an important part of the dynamics by looking only at linearizations around equilibrium: there are two additional speeds allowed for the shear waves that collapse onto the remaining two upon linearization, so that these additional velocities are not visible in the linearized analysis.

We presented two sets of conditions for causality, namely, conditions that are necessary and conditions that are sufficient. Further studies must be done to discover conditions that are necessary and sufficient, i.e., conditions 
that ensure the system to be causal if and only if they hold. This is an extremely challenging task given the complexity of the characteristic equation in the nonlinear problem.

Conformal limit.-To obtain some physical understanding about our nonlinear constraints, consider a conformal fluid [55], i.e., $\Pi=0, P=\varepsilon / 3, \delta_{\pi \pi}=4 \tau_{\pi} / 3$, with $\eta / s$ and $\tau_{\pi} T$ being constants (here, $T \sim \varepsilon^{1 / 4}$ is the temperature and $s \sim T^{3}$ is the equilibrium entropy density). Assume, for simplicity, that all the other transport coefficients vanish (as in [82]). The necessary conditions in (4) then impose that $\Lambda_{a} /(\varepsilon+P) \geq-1+(\eta / s)\left(1 / \tau_{\pi} T\right)$, so none of the eigenvalues of $\pi_{\nu}^{\mu}$ can be too negative. Also, when $\Lambda_{a} /(\varepsilon+P)>-1+(\eta / s)\left(1 / \tau_{\pi} T\right)$, the eigenvalues are also limited from above since (4e) gives $\Lambda_{a} /(\varepsilon+P) \leq 1-\left(2 / \tau_{\pi} T\right)(\eta / s)$. Using typical values motivated by heavy-ion collision applications, $\eta / s=$ $1 /(4 \pi) \quad$ [83] and $\tau_{\pi} T=5 \eta / s$ [84], one then finds $-4 / 5<\Lambda_{a} /(\varepsilon+P) \leq 3 / 5$. This implies that the relative magnitude of the shear stress tensor, $\sqrt{\pi_{\mu \nu} \pi^{\mu \nu} /(\varepsilon+P)^{2}}$, cannot be arbitrarily large. Using a NS initial condition where $\pi_{\mu \nu} \sim-2 \eta \sigma_{\mu \nu}$ at the initial time $\tau_{0}$, the corresponding normalized eigenvalues would be parametrically given by $\Lambda_{a} /(\varepsilon+P) \sim(\eta / s) /\left[\tau_{0} T\left(\tau_{0}, \vec{x}\right)\right]$ (assuming $\sigma_{\mu \nu} \sim 1 / \tau_{0}$ in the initial state). Given that our conditions imply that, roughly, $\left|\Lambda_{a} /(\varepsilon+P)\right| \lesssim 1, \quad$ for $\tau_{0}=0.6 \mathrm{fm}$ and $\eta / s=1 /(4 \pi)$, causality issues will be found where $T\left(\tau_{0}, \vec{x}\right) \lesssim 30 \mathrm{MeV}$, which is below the typical values for the freeze-out temperature. However, in initial state models where the initialized $\pi_{\mu \nu} /(\varepsilon+P)$ is large and strongly deviates from NS, the estimate above does not apply, and causality violations may appear in hot regions of the plasma as well. A detailed numerical study is needed to assess the importance of our results to current simulations of heavy-ion collisions.

Conclusions. - In this Letter, we established, for the first time, that causality, in fact, holds for the full set of nonlinear equations in IS-like theories without the need for symmetry assumptions and in the presence of both shear and bulk viscosity. All our conditions are simple algebraic inequalities among the dynamical variables that can be easily checked in a given system or simulation. Previous attempts to go beyond the linear regime were restricted to $1+1$ dimensions [60] or assumed strong symmetry conditions [61,85], which, in practice, also corresponds to partial differential equations with only one spatial variable. Without such restrictions, the only other work where nonlinear causality has been shown for IS-like systems is [79], which only included bulk viscous effects. We have also studied the Cauchy problem for (1)-(2), establishing that it is well defined, so that it is meaningful to talk about solutions.

Prior to our work, unless a numerical code was specifically tailored to detect causality violations of the underlying equations, which typically is not a feature present in standard codes, one could only identify whether a numerical simulation of (1)-(2) violated causality if this caused (a) a breakdown of the simulation, (b) a manifestly spurious solution, or (c) clear nonphysical behavior. These constraints are all too weak, as we now explain. For illustration, consider $-\partial_{t}^{2} \psi+(1+\psi) \Delta \psi=0$, where $\Delta$ is the Laplacian. This is a nonlinear wave equation with (nonlinear) speed given by $\sqrt{1+\psi}$ for (For $\psi<-1$, the equation is no longer a wave equation, becoming elliptic, and it is a degenerate wave equation when $\psi=-1$.) $\psi>-1$. Indeed, the characteristics are given by $\xi_{0}= \pm \sqrt{1+\psi}|\vec{\xi}|$. Therefore, the solutions are not causal when $\psi>0$, but are causal for $-1<\psi \leq 0$. Nevertheless, the equation remains hyperbolic as long as $\psi>-1$. Standard hyperbolic theory (see, e.g., [86]) ensures that, given smooth initial data $\left.\psi\right|_{t=0}$ and $\left.\partial_{t} \psi\right|_{t=0}$, there exists a unique smooth solution defined for some time. So any numerical scheme that is able to track the unique solution will produce results in both the acausal and causal cases $\psi>0$ and $-1<\psi \leq 0$, respectively. This makes it extremely difficult to infer violations of causality using (a) or (b) as criteria. Exactly the same situation can happen in simulations of (1)-(2). We also note that linearizing the equation about the "equilibrium" $\psi=0$ gives $-\delta \psi_{t t}+\Delta \delta \psi=0$, which is always causal, reinforcing, again, the idea that causality cannot always be obtained from linearizations.

Criteria (c) has also limited applicability. First, there are different mechanisms that can produce nonphysical solutions. Thus, it is still important to understand whether unphysical behavior is being caused by causality violation or some other mechanism, such as running beyond the limit where the effective description is valid. Second, relativistic fluids in the far-from-equilibrium regime, such as the QGP, may exhibit unexpected behavior, so one needs to be careful to differentiate genuine exotic features from those that are consequences of running a simulation in a superluminal regime. This may be particularly relevant to heavyion simulations where the values of the fields drop extremely rapidly at the edges of the QGP at early times and in the cold or dilute regions of plasma where a rescaling of dissipative tensors has been employed [87-90]. Third, numerical simulations of relativistic fluids must be based on equations of motion that respect causality, a fundamental physical principle in relativity.

The results we presented here are an important step in addressing all these difficulties, as one can check if (A1)-(A3), (4), or (5) hold at any moment in numerical simulations [Comparing with the example of the equation for $\psi$ above, this would be similar to monitoring the value of $\sqrt{1+\psi}$ : if $\psi>1$, then the system is not causal, which is the analog of (5), whereas causality is guaranteed if $-1<\psi \leq 0$, which is the analog of (5).] since all the quantities involved in our inequalities can be readily extracted in numerical simulations [3]. We also note that 
our results apply, in particular, to the initial conditions, so (4) and (5) can be used to rule out initial conditions that violate causality or to select initial conditions for which causality holds. This can be particularly relevant to further constrain the physical assumptions behind the modeling of initial conditions in QGP simulations. There are many subtleties involved in numerically solving the IS equations, including possible violations of causality caused entirely as an artifact of the numerical simulation. Thus, it is important to distinguish between such numerically caused unphysical phenomena from true violation of causality of the underlying equations. Our new causality criteria can be instrumental in such analyses.

In sum, in this Letter, we established, for the first time in the literature, conditions to settle the longstanding questions concerning causality in Israel-Stewart theories in the nonlinear, far-from-equilibrium regime. As such, our general results provide the most stringent tests to date for determining the validity of relativistic fluid dynamic approaches in heavy-ion collisions, astrophysics, and cosmology.

M. M. D. is partially supported by a Sloan Research Fellowship provided by the Alfred P. Sloan Foundation, NSF Grant No. DMS-1812826, a Discovery Grant administered by Vanderbilt University, and by a Dean's Faculty Fellowship. V.H. was funded (full or in-part) by the University of Texas at San Antonio, Office of the Vice President for Research, Economic Development, and Knowledge Enterprise. V. H. acknowledges partial support by NSF Grants No. DMS-1614797 and No. DMS1810687. J.N. is partially supported by the U.S. Department of Energy, Office of Science, Office for Nuclear Physics under Award No. DE-SC0021301.

*fabio.bemfica@ect.ufrn.br †marcelo.disconzi@vanderbilt.edu *duynguyenvu.hoang@utsa.edu jn0508@illinois.edu maria_radosz@hotmail.com

[1] U. Heinz and R. Snellings, Annu. Rev. Nucl. Part. Sci. 63, 123 (2013).

[2] G. Gale, S. Jeon, and B. Schenke, Int. J. Mod. Phys. A 28, 1340011 (2013).

[3] P. Romatschke and U. Romatschke, Relativistic Fluid Dynamics In and Out of Equilibrium, Cambridge Monographs on Mathematical Physics (Cambridge University Press, Cambridge, England, 2019).

[4] B. Schenke, P. Tribedy, and R. Venugopalan, Phys. Rev. Lett. 108, 252301 (2012).

[5] H. Niemi and G. S. Denicol, arXiv:1404.7327.

[6] J. Noronha-Hostler, J. Noronha, and M. Gyulassy, Phys. Rev. C 93, 024909 (2016).

[7] M. Luzum and H. Petersen, J. Phys. G 41, 063102 (2014).

[8] P. Bozek, Phys. Rev. C 85, 014911 (2012).
[9] V. Khachatryan et al. (CMS Collaboration), Phys. Rev. Lett. 115, 012301 (2015).

[10] G. Aad et al. (ATLAS Collaboration), Phys. Rev. Lett. 116, 172301 (2016).

[11] V. Khachatryan et al. (CMS Collaboration), Phys. Lett. B 765, 193 (2017).

[12] R. D. Weller and P. Romatschke, Phys. Lett. B 774, 351 (2017).

[13] C. Aidala et al. (PHENIX Collaboration), Nat. Phys. 15, 214 (2019).

[14] S. Acharya et al. (ALICE Collaboration), Phys. Rev. Lett. 123, 142301 (2019).

[15] F. S. Bemfica, M. M. Disconzi, and J. Noronha, Phys. Rev. D 98, 104064 (2018).

[16] F. S. Bemfica, M. M. Disconzi, and J. Noronha, Phys. Rev. D 100, 104020 (2019).

[17] P. Kovtun, J. High Energy Phys. 10 (2019) 034.

[18] R. E. Hoult and K. Pavel, J. High Energy Phys. 06 (2020) 067.

[19] M. P. Heller, R. A. Janik, and P. Witaszczyk, Phys. Rev. Lett. 110, 211602 (2013).

[20] M. P. Heller and M. Spalinski, Phys. Rev. Lett. 115, 072501 (2015).

[21] A. Buchel, M. P. Heller, and J. Noronha, Phys. Rev. D 94, 106011 (2016).

[22] G. S. Denicol and J. Noronha, arXiv:1608.07869.

[23] M. P. Heller, A. Kurkela, M. Spalinski, and V. Svensson, Phys. Rev. D 97, 091503(R) (2018).

[24] P. Romatschke, Phys. Rev. Lett. 120, 012301 (2018).

[25] M. Spalinski, Phys. Lett. B 776, 468 (2018).

[26] M. Strickland, J. Noronha, and G. S. Denicol, Phys. Rev. D 97, 036020 (2018).

[27] P. Romatschke, J. High Energy Phys. 12 (2017) 079.

[28] W. Florkowski, M. P. Heller, and M. Spalinski, Rep. Prog. Phys. 81, 046001 (2018).

[29] G. S. Denicol and J. Noronha, Phys. Rev. D 97, 056021 (2018).

[30] A. Behtash, C. N. Cruz-Camacho, and M. Martinez, Phys. Rev. D 97, 044041 (2018).

[31] J.-P. Blaizot and L. Yan, Phys. Lett. B 780, 283 (2018).

[32] D. Almaalol and M. Strickland, Phys. Rev. C 97, 044911 (2018).

[33] G. S. Denicol and J. Noronha, Phys. Rev. D 99, 116004 (2019).

[34] K. Gallmeister, H. Niemi, C. Greiner, and D. H. Rischke, Phys. Rev. C 98, 024912 (2018).

[35] J. Casalderrey-Solana, C. P. Herzog, and M. Meiring, J. High Energy Phys. 01 (2019) 181.

[36] A. Behtash, C. N. Cruz-Camacho, S. Kamata, and M. Martinez, Phys. Lett. B 797, 134914 (2019).

[37] A. Behtash, S. Kamata, M. Martinez, and H. Shi, Phys. Rev. D 99, 116012 (2019).

[38] M. Strickland, J. High Energy Phys. 12 (2018) 128.

[39] S. Jaiswal, C. Chattopadhyay, A. Jaiswal, S. Pal, and U. Heinz, Phys. Rev. C 100, 034901 (2019).

[40] A. Kurkela, W. van der Schee, U. A. Wiedemann, and B. Wu, Phys. Rev. Lett. 124, 102301 (2020).

[41] G. Giacalone, A. Mazeliauskas, and S. Schlichting, Phys. Rev. Lett. 123, 262301 (2019).

[42] G. S. Denicol and J. Noronha, Phys. Rev. Lett. 124, 152301 (2020). 
[43] C. Chattopadhyay and U. W. Heinz, Phys. Lett. B 801, 135158 (2020).

[44] D. Almaalol, A. Kurkela, and M. Strickland, Phys. Rev. Lett. 125, 122302 (2020).

[45] A. Das, W. Florkowski, J. Noronha, and R. Ryblewski, Phys. Lett. B 806, 135525 (2020).

[46] W. Israel, Ann. Phys. (N.Y.) 100, 310 (1976).

[47] W. Israel and J. M. Stewart, Ann. Phys. (N.Y.) 118, 341 (1979).

[48] I. Mueller, Z. Phys. 198, 329 (1967).

[49] G. Pichon, Ann. Inst. H. Poincaré Sect. A (N.S.) 2, 21 (1965).

[50] W. A. Hiscock and L. Lindblom, Phys. Rev. D 31, 725 (1985).

[51] C. Eckart, Phys. Rev. 58, 919 (1940).

[52] L. D. Landau and E. M. Lifshitz, Fluid Mechanics_Volume 6 (Course of Theoretical Physics), 2nd ed. (ButterworthHeinemann, London, 1987).

[53] D. Jou, J. Casas-Vazsquez, and G. Lebon, Extended Irreversible Thermodynamics, 3rd ed. (Springer, New York, 2001).

[54] I. Mueller and T. Ruggeri, Rational Extended Thermodynamics (Springer, New York, 1998).

[55] R. Baier, P. Romatschke, D. T. Son, A. O. Starinets, and M. A. Stephanov, J. High Energy Phys. 04 (2008) 100.

[56] G. S. Denicol, H. Niemi, E. Molnar, and D. H. Rischke, Phys. Rev. D 85, 114047 (2012); 91, 039902(E) (2015).

[57] S. Ryu, J.-F. Paquet, C. Shen, G. Denicol, B. Schenke, S. Jeon, and C. Gale, Phys. Rev. C 97, 034910 (2018).

[58] W. A. Hiscock and L. Lindblom, Ann. Phys. (N.Y.) 151, 466 (1983).

[59] T. S. Olson, Ann. Phys. (N.Y.) 199, 18 (1990).

[60] G. S. Denicol, T. Kodama, T. Koide, and P. Mota, J. Phys. G 35, 115102 (2008).

[61] S. Pu, T. Koide, and D. H. Rischke, Phys. Rev. D 81, 114039 (2010).

[62] R. M. Wald, General Relativity (University of Chicago Press, Chicago, 2010).

[63] Y. Choquet-Bruhat, General Relativity and the Einstein Equations (Oxford University Press, New York, 2009).

[64] J. M. Stewart, Proc. R. Soc. A 357, 59 (1977).

[65] W. Israel and J. M. Stewart, Proc. R. Soc. A 365, 43 (1979).

[66] W. Israel and J. M. Stewart, Phys. Lett. A 58, 213 (1976).

[67] I. Brevik, O. Gron, J. de Haro, S. D. Odintsov, and E. N. Saridakis, Int. J. Mod. Phys. D 26, 1730024 (2017).

[68] G. S. Denicol, S. Jeon, and C. Gale, Phys. Rev. C 90, 024912 (2014).

[69] S. I. Finazzo, R. Rougemont, H. Marrochio, and J. Noronha, J. High Energy Phys. 02 (2015) 051.
[70] M. M. Disconzi, Nonlinearity 27, 1915 (2014).

[71] T. Kato, Arch. Ration. Mech. Anal. 58, 181 (1975).

[72] A. E. Fischer and J. E. Marsden, Commun. Math. Phys. 28, 1 (1972).

[73] L. C. Evans, Partial Differential Equations, 2nd ed., Vol. 19 of Graduate Studies in Mathematics (American Mathematical Society, Providence, RI, 2010).

[74] A. Majda, Compressible Fluid Flow and Systems of Conservation Laws in Several Space Variables, Vol. 53 of Applied Mathematical Sciences (Springer-Verlag, New York, 1984).

[75] J. Leray, Hyperbolic Differential Equations (The Institute for Advanced Study, Princeton, N.J., 1953).

[76] M. M. Disconzi and J. Speck, Ann. Inst. Henri Poincaré 20, 2173 (2019).

[77] C. Courant and D. Hilbert, Methods of Mathematical Physics, 1st ed., Vol. 2 (John Wiley \& Sons, Inc., New York, 1991).

[78] See Supplemental Material at http://link.aps.org/ supplemental/10.1103/PhysRevLett.126.222301 for the details concerning the calculation of the characteristics and the corresponding proofs of the necessary and sufficient conditions presented in the main text.

[79] F. S. Bemfica, M. M. Disconzi, and J. Noronha, Phys. Rev. Lett. 122, 221602 (2019).

[80] G. S. Denicol and J. Noronha, Nucl. Phys. A1005, 121748 (2021).

[81] M. G. Alford, L. Bovard, M. Hanauske, L. Rezzolla, and K. Schwenzer, Phys. Rev. Lett. 120, 041101 (2018).

[82] H. Marrochio, J. Noronha, G. S. Denicol, M. Luzum, S. Jeon, and C. Gale, Phys. Rev. C 91, 014903 (2015).

[83] P. K. Kovtun, D. T. Son, and A. O. Starinets, Phys. Rev. Lett. 94, 111601 (2005).

[84] G. S. Denicol, J. Noronha, H. Niemi, and D. H. Rischke, Phys. Rev. D 83, 074019 (2011).

[85] S. Floerchinger and E. Grossi, J. High Energy Phys. 08 (2018) 186.

[86] C. D. Sogge, Lectures on Non-Linear Wave Equations, 2nd ed. (International Press, Boston, MA, 2008).

[87] B. Schenke, S. Jeon, and C. Gale, Phys. Rev. C 85, 024901 (2012).

[88] P. Bozek, Phys. Rev. C 85, 034901 (2012).

[89] C. Shen, Z. Qiu, H. Song, J. Bernhard, S. Bass, and U. Heinz, Comput. Phys. Commun. 199, 61 (2016).

[90] D. Bazow, U. W. Heinz, and M. Strickland, Comput. Phys. Commun. 225, 92 (2018). 